\title{
Partial dielectric behavior of the Mo electron spectrum as an effect of Van Hove singularities
}

\author{
T.A. Ignatyeva \\ National Science Center "Kharkiv Institute of Physics and Technology", \\ 1, Academichna str., 61108 Kharkiv, Ukraine \\ E-mail: taignatieva@mail.ru
}

\begin{abstract}
The investigation shows that the specific conductivity of Mo sharply decreases exponentially under the temperature influence within the range from $\sim 20$ to $\sim 60 \mathrm{~K}$ or under the Re impurity influence in the concentration range up to $3-4$ at.\% and then transforms into the power dependence. Noted there are two singularities in the Mo specific conductivity, namely, an exponential conductivity change within the small energy range and the presence of a threshold energy value equivalent to $\sim 50 \mathrm{~K}$, which can be related to the mobility edge for localized electron states at the spectrum edge in the vicinity of the critical energy $\varepsilon_{C 1}$ (arising of a small-size electron lens). The identity in the behavior of Mo specific conductivity change, independently on the external parameter influencing on the Fermi level position relatively to the critical points of the electron spectrum, is shown. This fact permits to assume that the singularities under consideration can be related to the partial dielectric behavior of the electron spectrum, depending on the Fermi level position relatively to the critical energies
\end{abstract}

Keywords: electron spectrum, dielectric behavior, critical energies, specific conductivity, spectrum edge, localized states, electron state density.

Manuscript received 25.06.11; revised manuscript received 30.08.11; accepted for publication 14.09.11; published online 30.11.11.

\section{Introduction}

As own from literature, the Van Hove singularities [1] lead to the electron topological transitions (ETT) $[2,3]$ under conditions of the external action on the position of Fermi level $\varepsilon_{\mathrm{F}}$ relatively to these singularities $\varepsilon_{C}$ [ $4-7$ ]. In the case of electron topological transitions of a 2.5 kind, the singularities arise in the electron states density $\delta v(\varepsilon) \sim \sqrt{\varepsilon_{C}-\varepsilon_{\mathrm{F}}}$ (for free electrons). Moreover, a characteristic singularity is the change in the electron motion dynamics, when the Fermi level traverses the critical point of the electron spectrum.

The present paper considers ETT caused by the small-size electron group arising, when the Fermi level traverses the bottom of a band, not occupied before, then the electron velocity reduces to zero and the effective mass is increasing. At the same time, it is known that when the Fermi level traverses the zone bottom, the states of electrons at the spectrum edge are localized with the characteristic signs of metal-dielectric transition [8], namely, the appearance of $\delta$-like peaks in the electron state density, corresponding to the quasidiscrete spectrum to some mobility threshold, during electron delocalization.

The metal-dielectric transitions were considered for the most part for semiconductors. In the case of electron topological transitions in metals (appearance of a smallsize electron group) there are, simultaneously, signs of two types of transitions with characteristic singularities in the electron state densities for the ETT and the metaldielectric transition. 
Simultaneously with the singularity $\delta v(\varepsilon)$ $\sim \sqrt{\varepsilon_{C}-\varepsilon_{\mathrm{F}}}$ for free electrons, there arising are $\delta$-like peaks of the electron state density at the spectrum edge inherent to the localized electron states. Singularities in the electron motion dynamics during such electron transitions lead to new mechanisms of charge transfer with participation of phonons, i.e., to the umklapp processes [9-11] and to additional mechanisms in the scattering processes.

So, under the condition $\varepsilon_{\mathrm{F}}-\varepsilon_{C}=0$ the singularities arise in the electron state densities, being inherent to two types of transitions, namely, ETT and metal-dielectric transition. Before fulfilling this condition, the Fermi level changes its position relatively to the critical energy that results in the change of the energy gap under the external actions. It should lead to the exponential dependence of the specific resistance, characteristic for the metal-dielectric transition, on the activation energy and the gap size $\Delta \varepsilon$, in this case it is the gap between the Fermi level and the critical electron energy.

Note that both of transitions have a common nature. In both cases, the Fermi level passes through the spectrum edge (zone bottom). This fact allows us to conclude that the electron-topological transition in metals should be accompanied by the metal-dielectric transition in the direction $\varepsilon(\kappa)$ relatively to the Brillouin zone, where there is a narrow gap between the Fermi level $\varepsilon_{\mathrm{F}}$ and the critical point of electron spectrum $\varepsilon_{C}$ (Brillouin zone boundary).

Under the external actions (pressure, impurity, temperature), one can create conditions for realization of two interrelated electron transitions having the features of partial dielectric behavior of the electron spectrum in the direction $\varepsilon(\kappa)$ where the ETT occurs.

For the first time, two types of singularities in the electron state densities have been found by studying the ETT under external actions in the Mo-Re alloys [5-7]. The dependences of the superconducting transition temperature $T_{C}$ under pressure were measured with a high sensitivity $\left(10^{-3} \mathrm{~K}\right)[5,6]$. The thermal e.m.f. $\alpha(T)$ for different concentrations of $\mathrm{Mo}-\mathrm{Re}$ alloys within the temperature range from 0 to $10 \mathrm{~K}$ were measured with the sensitivity $10^{-13} \mathrm{~V}[7]$.

Due to the high sensitivity of measurements for the $T_{C}(P)$ and $\alpha / T(T)$ dependences within the temperature range from 0 to $10 \mathrm{~K}$ in the $\mathrm{Mo}-\mathrm{Re}$ alloys, simultaneously with a smooth nonlinearity corresponding to the ETT singularity $\delta v(\varepsilon) \sim \sqrt{\varepsilon_{C}-\varepsilon_{\mathrm{F}}}$, the "oscillations" having low amplitudes were observed. These "oscillations" were assumed to be related with the Fermi level transition through the local levels of the quasi-discrete spectrum at the edge of a new zone $\left(\varepsilon_{C 2}\right)$, corresponding to the appearance of a new Fermi surface cavity with 10 at $\% \operatorname{Re}[12]$. The ETT in these alloys was determined as an extreme of derivatives of the investigated characteristics by the external parameter (pressure, impurity, temperature), being changing the gap $\varepsilon_{C}-\varepsilon_{\mathrm{F}}$.

Later the attention was given to the fact that singularities in the temperature dependence of the specific resistance of pure Mo also can be considered as appearance of quasi-local levels of the electron spectra due to the closeness of $\varepsilon_{\mathrm{F}}$ to the critical point $\varepsilon_{C 1}$ below the Fermi level belonging in Mo to the small-size electron lens [13]. In this case, the temperature is considered as a parameter exerting the influence on the Fermi level position relatively to the critical energy $\varepsilon_{C 1}$.

This paper presents the consideration on a macroscopic sharp decrease of the Mo specific conductivity (by the exponent) in the dependence $1 / \rho(T)$ within the narrow temperature range from $\sim 20$ up to $\sim 60 \mathrm{~K}$ and in the dependence $1 / \rho(C)$ within the range of rhenium impurity concentrations to $\sim 3-4$ at. $\%$ at $10 \mathrm{~K}$.

A comparison is made between the experimental results [14-16] on singularities in the temperature and concentration dependences of the specific conductivity for Mo, which can be related to the partial dielectric behavior of the Mo electron spectrum due to the closeness $\varepsilon_{C 1}$ of the small-size electron lens in Mo to the Fermi level.

\section{Experimental results}

The present paper gives the results of experimental investigations [14-16] on the temperature dependence of the specific resistance $\rho(T)$ and specific conductivity $1 / \rho(T)$ in a wide temperature range for Mo with the residual resistance $R_{4.2} / R_{300}=4.5 \times 10^{-4}$, as well as the concentration dependence $\rho(C)$ and $1 / \rho(C)$ of Mo-Re alloys within the solid solution at $10 \mathrm{~K}$.

Making the samples and measurement techniques are described in [6,7], where the results have been considered only with account of the singularity $\varepsilon_{C 1}$ in the Mo electron spectrum above the Fermi level that leads to the electron-topological transition under the $\mathrm{Re}$ impurity influence, i.e. to the formation of a new Fermi surface cavity when the Re concentration reaches $\sim 10$ at. $\%$.

In this paper, we pay attention to other singularities in the specific resistance of Mo related with the presence of a critical point in the Mo electron spectrum below the Fermi level $\varepsilon_{C 1}$, belonging to the available small-size electron lens. When the Fermi level moves in the narrow range of energies $\varepsilon_{C}-\varepsilon_{\mathrm{F}}$, one can observe singularities in the specific resistance of Mo depending on the Fermi level position relatively to $\varepsilon_{C 1}$ The data presented consider the Fermi level position being varied by the 
temperature for Mo above $20 \mathrm{~K}$ and or by the $\mathrm{Re}$ impurity at a constant temperature of $10 \mathrm{~K}$.

The experimental results on the dependences of changes in the specific resistance and conductivity of Mo under the temperature influence in the wide temperature range and under the Re impurity influence for Mo-Re alloys in the region of solid solution are given in Figs. 1a,b and 2 a,b, respectively.

The results presented in Fig. 1a demonstrate two characteristic singularities which manifest themselves in the temperature derivatives of specific resistance in the form of extremes within the temperature ranges from 20 to $30 \mathrm{~K}$ and from 150 to $200 \mathrm{~K}$ (Fig. 1a, curve 2). These singularities are related with electron transitions in Mo caused by the temperature change (see details in [13]).

Fig. 1b shows the specific conductivity of Mo as a function of temperature within the range $1.5-300 \mathrm{~K}$, and the inserts in Fig. 1b show the specific conductivity of Mo as a function of temperature in different temperature ranges. Here, attention should be given to the change in the functional dependence $1 / \rho(T)$, i.e. the transition from the exponential dependence describing the sharp change of the conductivity to the power dependence during further temperature increase.

Fig. 2a, b shows the Re impurity influence on the specific resistance (Fig. 2a) and on the specific conductivity (Fig. 2b). Here, also the exponential
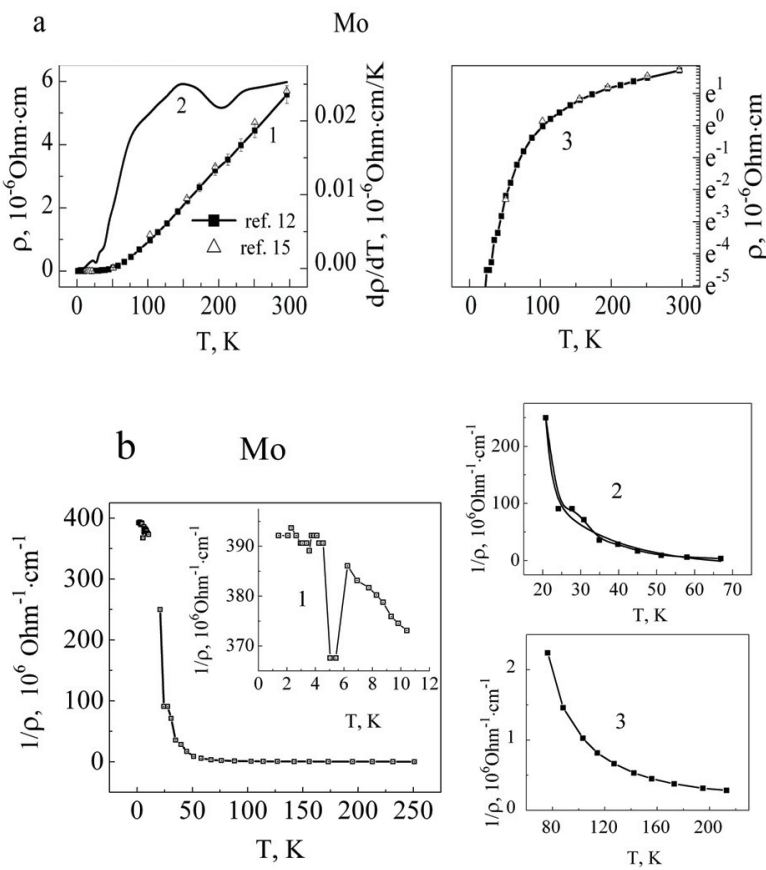

Fig. 1. a) Mo specific resistance (linear 1 and logarithmic 3 scales) and the derivative versus temperature (2) for Mo; b) Mo specific conductivity versus the temperature within the range from 1.5 to $300 \mathrm{~K}$. The inserts 1,2 , and 3 show different temperature ranges. dependence variation $1 / \rho(C)$ in the concentration range to 3-4 at.\% Re changes to the power dependence variation during further concentration increase.

From the above-mentioned results, one can see that the changes in the specific resistance and the specific conductivity are identical as the Fermi level rises, and no difference which of external parameters influences on the Fermi level position (in our case, temperature or impurity). The distinction is a sharp exponential decrease of the conductivity for pure Mo within the narrow temperature range from 30 to $60 \mathrm{~K}$ and within the narrow concentration range, for $\mathrm{Mo}-\mathrm{Re}$ alloys to $3-4$ at. $\%$ at $10 \mathrm{~K}$.

\section{Discussion on results}

To analyze the results obtained, let us reduce them to the single parameters, namely, the relative change of the electron state density $\frac{\delta v}{v_{0}}(\varepsilon)$ under the temperature and impurity concentration influence on the electron spectrum structure.

From the data presented, this value is obtained using the following way

$$
\begin{aligned}
& \left(\rho-\rho_{0}\right) \sim \frac{1}{v_{0}+\delta v}-\frac{1}{v_{0}} \sim-\frac{\delta v}{\left(v_{0}+\delta v\right) v_{0}}, \\
& \frac{\left(\rho-\rho_{0}\right)}{\rho}=-\frac{\delta v}{v_{0}},
\end{aligned}
$$

where $\rho_{0}$ and $v_{0}$ do not depend on the changes in the electron spectrum and are determined by the initial state of the sample, $\rho$ is the specific resistance at different temperatures and composition of the sample with the electron state density $\left(v_{0}+\delta v\right)$, where $\delta v_{0}$ is the addition to the electron state density depending on the external parameter influence and on the electron transition nature.

In the paper [13], one considers the dependence $\left(\rho-\rho_{0}\right) / \rho$ as a function at Mo, temperature in the wide temperature range with a detailed discussion of the changes in the Mo electron spectrum under the influence of temperature in the different temperature ranges.

In our paper, we compare the dependence $\left(\rho-\rho_{0}\right) / \rho$ of Mo as a function temperature exceeding $30 \mathrm{~K}$ with such dependence as a function of Re impurity concentration in Mo. The choice of this temperature range is determined by the following reasons: for comparison of the influence of different external parameters (impurity and temperature) on the Mo electron spectrum, it is necessary to have an identity of the Mo electron spectrum, beginning from which the different external parameters (impurity and temperature), rising the Fermi level, exert the influence on the further change in the electron spectrum. 


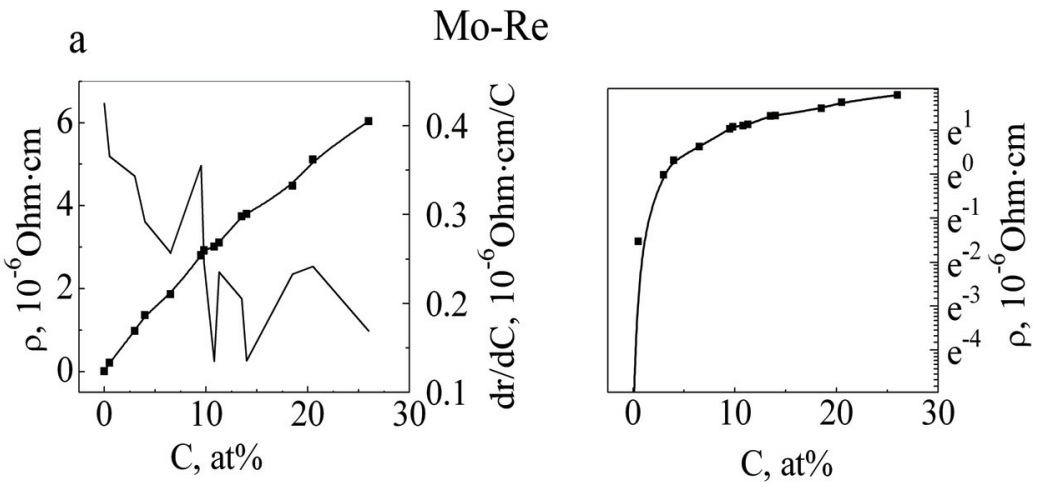

$\mathrm{b}$

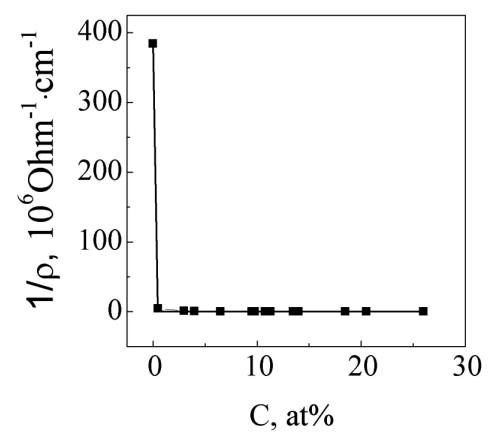

Mo-Re
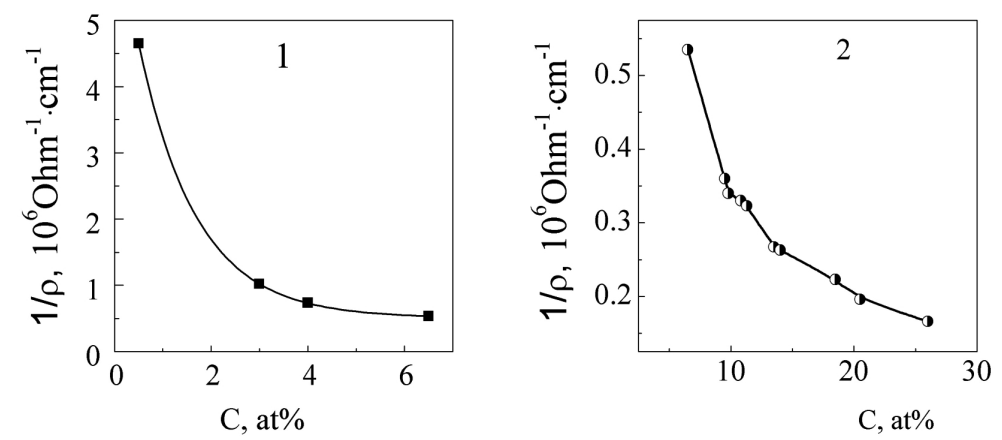

Fig. 2. a) Mo-Re specific resistance (in the linear and logarithmic scales) and the derivative versus the concentration; b) Mo - Re specific conductivity versus the concentration within the range from 0 to 25 at.\%Re for Mo - Re. The inserts 1 and 2 show different concentration ranges.

Mo
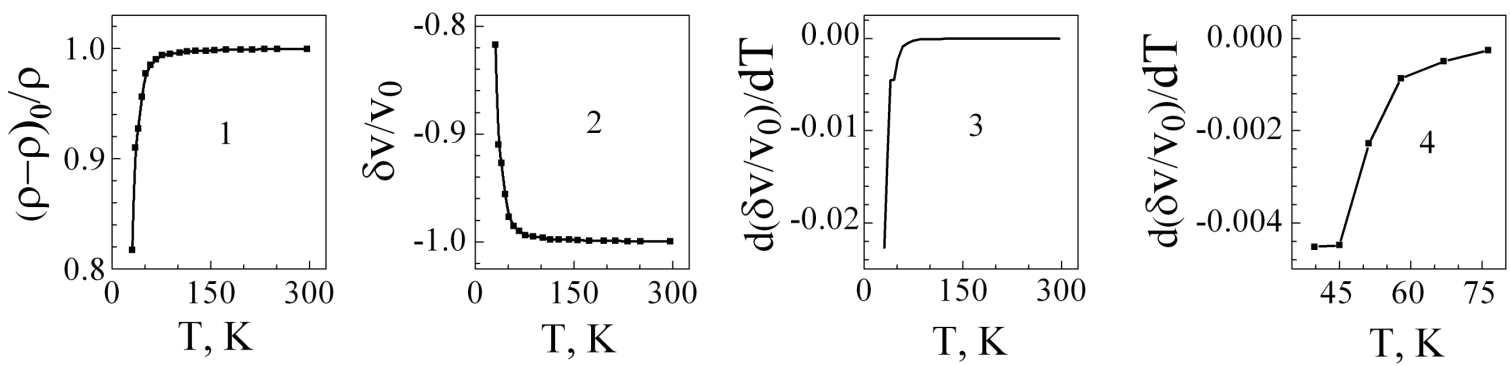

Fig. 3. Dependences $\left(\frac{\rho-\rho_{0}}{\rho}\right)$ of temperature function (1), of electron state density changes $\frac{\delta v}{v_{0}}(T)(2), d \frac{\delta v}{v_{0}} / d T(T)(3,4)$ for Mo.

According to our notions [13], at temperatures lower than $10 \mathrm{~K}$ the electron lens in Mo $\left(\varepsilon_{C 1}\right)$ vanishes, due to the Fermi level lowering in the case of the lattice expansion, and at $T>10 \mathrm{~K}$ it recovers within the temperature range 20 to $30 \mathrm{~K}$, when the smearing of $k T$ compensates the Fermi level drop, and the temperature influence on the Fermi level position, during the further temperature increase, reduces to the Fermi energy displacement upwards.

In other words, we compare the behavior of the specific conductivity under conditions that the Fermi level moves upwards under the influence of different parameters (temperature, Fig. 3, and impurity, Fig. 4, beginning from the same starting conditions, by the Fermi level position, relatively to the critical energies of the Mo electron spectrum).

Figs. $3 \mathrm{~b}$ and $3 \mathrm{c}$ represent the dependences $\frac{\delta v}{v_{0}}(T)$ and the temperature derivative of this value for Mo. Figs. $4 \mathrm{~b}$ and $4 \mathrm{c}$ present the dependences $\frac{\delta v}{v_{0}}(C)$ and the concentration derivative of this value for $\mathrm{Mo}-\mathrm{Re}$.

Comparison of Figs. 3 and 4 shows the identity of the changes in the electron state density when the Fermi level rises independently on the choice of the external 

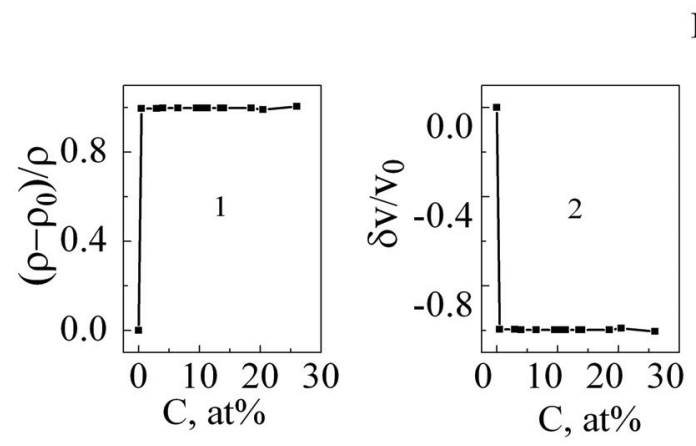

Mo-Re
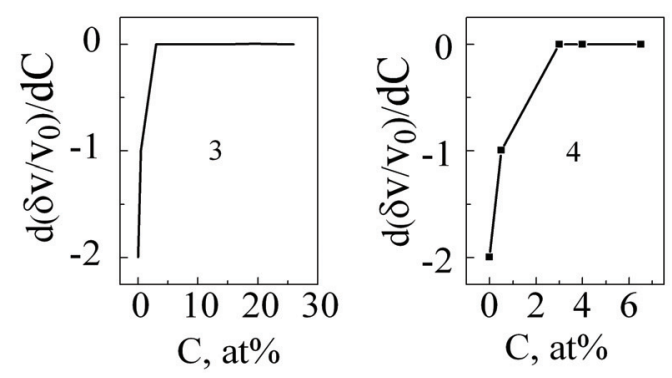

Fig. 4. Dependences $\left(\frac{\rho-\rho_{0}}{\rho}\right)$ of concentration function (1), of electron state density changes $\frac{\delta v}{v_{0}}(C) \quad(2), d \frac{\delta v}{v_{0}} / d C(C)$

$(3,4)$ for Mo - Re.

parameter displacing the Fermi level upwards: this is the sharp change of the characteristics under consideration within the temperature range from 30 to $60 \mathrm{~K}$ and in the range of concentrations to $\sim 3-4$ at. $\%$ with a subsequent slight changing during further increase either of the temperature or the impurity concentration.

Let us compare these results with the fine structure of the Mo electron spectrum [18] shown in Fig. 5.

One can see from the calculation of $\varepsilon(\kappa)$ for Mo that there are two gaps: $\varepsilon_{\mathrm{F}}-\varepsilon_{C 1}$ from the zone bottom in the direction $\Gamma \mathrm{H}$ to the Fermi level that corresponds to the small-size electron lens of Mo and $\varepsilon_{C 2}-\varepsilon_{\mathrm{F}}$ in the direction HN from the Fermi level to the empty zone bottom. If the Fermi level changes, then ETT are possible - the lens vanishes if $\varepsilon_{\mathrm{F}}$ decreases, and the new Fermi cavity take place if $\varepsilon_{\mathrm{F}}$ reaches $\varepsilon_{C 2}$.

In our case, the sharp change in the conductivity under the influence of temperature or Re impurity is not related with ETT at $\varepsilon_{C 2}$.

When there is 10 at.\% Re in Mo, the Fermi level rises within the range from 30 to $60 \mathrm{~K}$, and in $\mathrm{Mo}-\mathrm{Re}$ the concentration changes to $3-4$ at.\% the small-size electron lens increases in the direction $\Gamma H$ of the Brillouin zone and the gap $\varepsilon_{C 2}-\varepsilon_{\mathrm{F}}$ decreases.

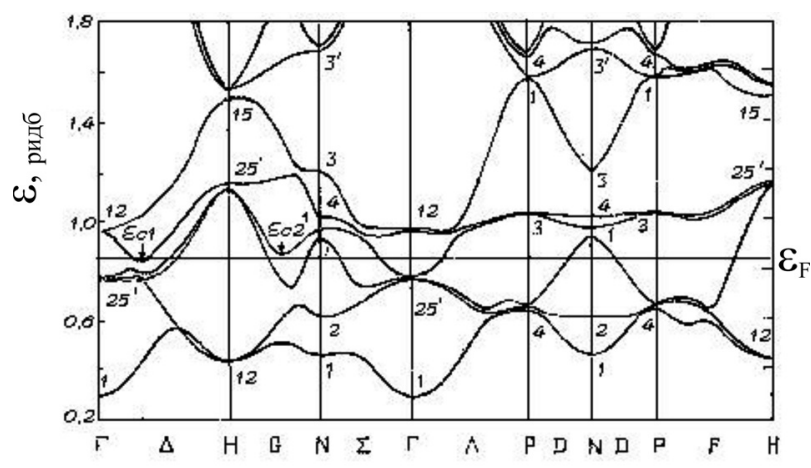

Fig. 5. Theoretical calculations of the Mo electron spectrum [18]. $\varepsilon_{C 1}$ is the electron lens nucleation; $\varepsilon_{C 2}$ is the empty zone bottom equivalent to the critical energy at which a new electron group arises under the influence of $\sim 10$ at. $\%$ Re.
One can see from the calculation of $\varepsilon(\kappa)$ for Mo that there are two gaps: $\varepsilon_{\mathrm{F}}-\varepsilon_{C 1}$ from the zone bottom in Mo the Fermi level rises within the range from 30 to $60 \mathrm{~K}$, and in $\mathrm{Mo}-\mathrm{Re}$ the concentration changes to $3-4$ at. $\%$, the small-size electron lens increases in the direction $\Gamma \mathrm{H}$ of the Brillouin zone and the gap $\varepsilon_{C 2}-\varepsilon_{\mathrm{F}}$ decreases.

Here, we should note two features in the electron spectrum change: 1) electron localization in Mo at the spectrum edge (for the lens it is equivalent to the zone bottom $\varepsilon_{C 1}$ ) and 2) additional conditions for the electron localization created by the $\mathrm{Re}$ addition (low concentrations) [19]. In both cases, the Fermi level moves in the energy gap $\varepsilon_{C 2}-\varepsilon_{\mathrm{F}}$ and changes its value.

In Fig. 6, given in more details are the temperature dependences of the characteristics under study for Mo at a temperature higher than $20 \mathrm{~K}$. It is seen that after the sharp increase of $\frac{\delta v}{v_{0}}(T)$, the decrease takes place within the range 30 to $60 \mathrm{~K}$.

The temperature derivatives in the range higher than $30 \mathrm{~K}$ are depicted in Fig. 7a, and the concentration derivatives up to $3-4$ at. $\%$ are in Fig. $7 b$. Under the temperature and impurity influence, these derivatives are increased in the identical manner.

Thus, singularities of conductivity in pure Mo can be related to the lens and the presence of localized electrons at the spectrum edge (zone bottom), when there is a nucleation (recovery) of the lens $\left(\varepsilon_{C 1}\right)$ at $\sim 30 \mathrm{~K}$. The influence of impurity and temperature on the electron spectrum is in correspondence with the different efficiency according to the rate of Fermi energy change under the influence of these parameters. From the data of $[6,7] \quad \frac{\partial \varepsilon}{\partial C}=\frac{\varepsilon_{\mathrm{F}}-\varepsilon_{C}}{C_{C}}=17 \times 10^{-4} \frac{\mathrm{eV}}{\text { at. } \%} \cdot$ Comparison of $\frac{\partial \varepsilon}{\partial T} \sim 10^{-4} \mathrm{eV} / \mathrm{K}$ and $\frac{\partial \varepsilon}{\partial C}$ shows that the influence of 1 at.\% $\operatorname{Re}$ on $\varepsilon_{\mathrm{F}}$ in Mo is equivalent to the temperature of $\sim 17 \mathrm{~K}$. Then at a temperature from 30 to $60 \mathrm{~K}$ and with the impurity concentration of $3-4$ at.\% Re, the 


\section{Mo}
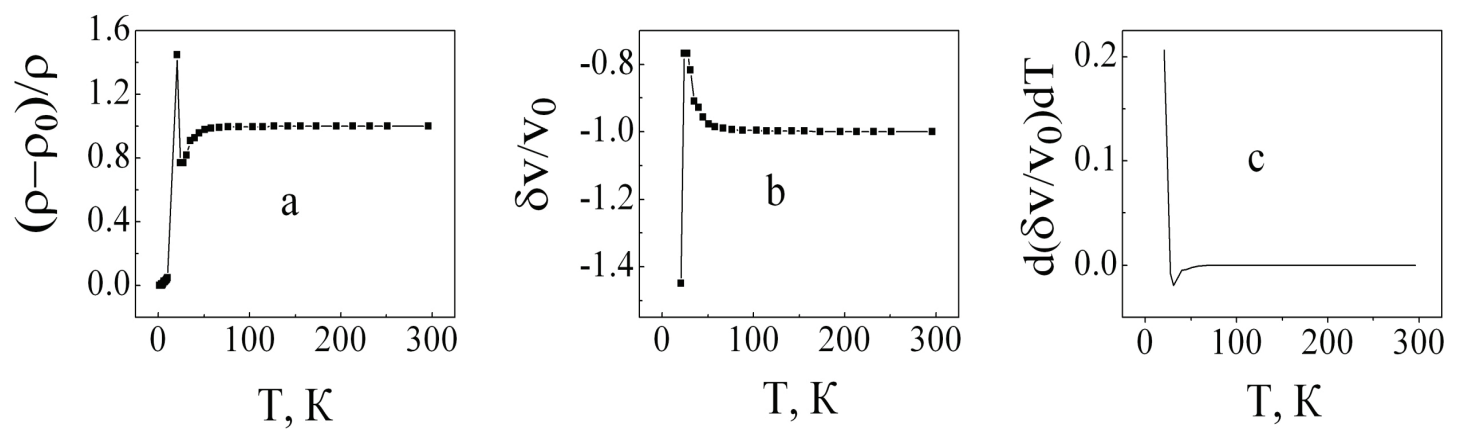

Fig. 6. The temperature dependence for Mo above $20 \mathrm{~K}: \frac{\rho-\rho_{0}}{\rho}(T), \frac{\delta v}{v_{0}}(T), d \frac{\delta v}{v_{0}} / d T(T)$.

Mo

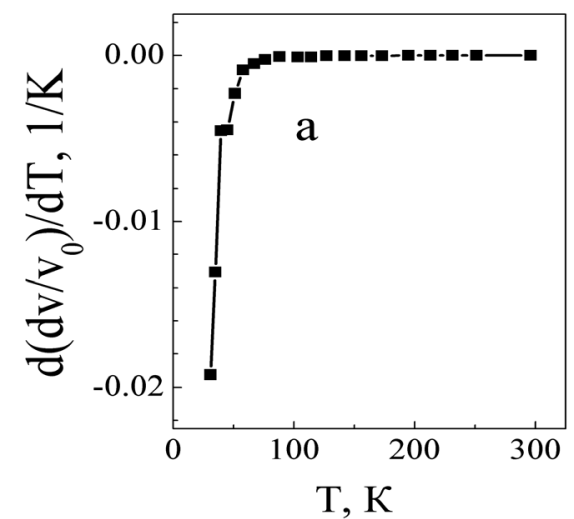

Mo-Re

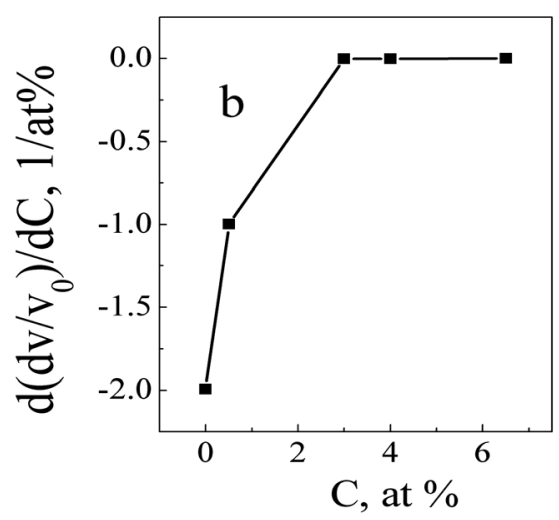

Fig. 7. a) The temperature derivative $d \frac{\delta v}{v_{0}} / d T(T)$ above $30 \mathrm{~K}$; b) the concentration derivative $d \frac{\delta v}{v_{0}} / d C(C)$.

Fermi level passes the same energy range to the threshold value, when the conductivity character changes from the exponential to the power one. It can be explained by the presence of a mobility edge that is characteristic for the metal-dielectric transition above which electrons delocalize. Comparison of the impurity and temperature influence on the change in the electron state density also can be made using the experimental data given below in Table.

\begin{tabular}{|c|c|c|c|}
\hline$C, \%$ & $d \frac{\delta v}{v_{0}} / d C$ & $T, \mathrm{~K}$ & $d \frac{\delta v}{v_{0}} / d T$ \\
\hline 0.5 & -0.9968 & 24.1 & 0.20667 \\
& & 27 & 0.10333 \\
& & 30.8 & -0.0079 \\
& & 34 & -0.01924 \\
& & 39.8 & -0.000452 \\
3 & -0.0008 & 51.2 & -0.00448 \\
4 & -0.00112 & 58 & -0.00228 \\
6.5 & & & \\
\hline
\end{tabular}

The data shows that under the influence of temperature near $50 \mathrm{~K}$, which is equivalent to the influence of 3-4 at.\% Re, there occurs the electron spectrum rearrangement not concerned to ETT but related to the same critical points of the electron spectrum, which causes the change in the Fermi surface topology when $\varepsilon_{\mathrm{F}}-\varepsilon_{C}=0$.

So, it can be assumed that the temperature of $\sim 50 \mathrm{~K}$ is an equivalent of the mobility edge energy for electrons localized at the spectrum edge or at the zone bottom.

\section{Conclusions}

1. In transition metals, besides a continuous electron spectrum, a discrete spectrum can exist, which is related with the electron localization due to the presence of small cavities of the Fermi surface, for which low-energy gaps between the Fermi energy and the critical energy, where a small-size electron group nucleates, are characteristic. The critical energy is simultaneously a zone bottom or an electron spectrum edge, where electrons are 
localized. As the Fermi energy increases, the number of localized states increases, too, up to the mobility edge, i.e. to the energy at which electrons are localized.

2. Our investigations show that in Mo, independently of the choice of parameters exerting the influence on the Fermi level position relatively to the electron spectrum critical energy $\varepsilon_{C 2}$ in the direction $\Gamma \mathrm{H}$ of the Brillouin zone, the singularities in the specific conductivity are identical. The specific conductivity behavior depends only on the energy range that the Fermi level passes from the critical point to the mobility edge under influence of external parameters (temperature or impurity).

3. The mobility edge in Mo equivalent by the energy to $\sim 50 \mathrm{~K}$ is determined. This temperature corresponds to the sharp change in the specific conductivity as a function of external parameters changing the Fermi level position. In the case under consideration, the matter concerns the partial dielectric behavior of the electron spectrum that leads to the exponential dependence of the Mo specific conductivity on the narrow energy range limited by the mobility threshold characteristic for metal-dielectric transitions.

\section{References}

1. 1. L. Van Hove, The occurrence of singularities in the elastic frequency distribution of a crystal // Phys. Rev. 89(6), p. 1189-1193 (1953).

2. I.M. Lifshits, On anomalities in electron characteristics of metals within the range of high pressures // Zhurnal experimental. teoret. fiziki, 38(5), p. 1569-1576 (1960), in Russian.

3. V.I. Makarov, V.G. Baryakhtar, On anomalities of superconductive transition temperature under pressure // Zhurnal experimental. teoret. fiziki, 48(6), p. 1717-1722 (1965), in Russian.

4. V.G. Baryakhtar, V.V. Gann, V.I. Makarov, T.A. Ignatyeva, Influence of changes in topology of the Fermi surface on superconductive properties // Zhurnal experimental. teoret. fiziki, 62(3), p. 11181128 (1972), in Russian.

5. T.A. Ignatyeva, Yu.A. Cherevan', On the features of changes in temperature of superconductive transition under pressure in Mo-Re solid solutions // Pis'ma v Zhurnal experimental. teoret. fiziki, 31(7), p. 389-392 (1980), in Russian.

6. T.A. Ignatyeva, V.V. Gann, A.N. Velikodnyi, Investigations of the electron-topological transition in superconductive alloys Mo-Re, Mo-Re-Nb // Fizika nizkikh temperatur, 20(11), p. 1133-1141 (1994), in Russian.
7. T.A. Ignatyeva, A.N. Velikodnyi, Thermo-e.m.f. features in Mo-Re, Mo-Re-Nb alloys and electrontopological transition in this systems // Fizika nizkikh temperatur, 28(6), p. 569-579 (2002), in Russian.

8. N.F. Mott, Transitions Metal-Insulator. Nauka, Moscow, 1979 (in Russian).

9. N.N. Bychkova, A.I. Kopeliovich, On the temperature dependence of metal electroresistance in a 'dirty' limit // Fizika nizkikh temperatur, 3(4), p. 458-467 (1977), in Russian.

10. Yu.M. Kogan, A.P. Zhernov, On the nature of nonlinear concentration dependence of resistance for metals with impurities // Zhurnal experimental. teoret. fiziki, 60(5), p. 1832-1844 (1971), in Russian.

11. N.V. Zavaritskii, A.I. Kopeliovich, V.I. Makarov, A.A. Yurgens, Electron-phonon interaction and topological features in the thermo-e.m.f. of metals // Zhurnal experimental. teoret. fiziki, 94(6), p. 344357 (1988), in Russian.

12. T.A. Ignatyeva, On localization of electrons under the electron-topological transition in Mo-Re alloys // Fizika tverdogo tela, 49(3), p. 389-397 (2007), in Russian.

13. T.A. Ignatyeva, Influence of Van Hove singularities on the temperature dependence of Mo resistivity // Metallofizika i noveishie tekhnologii, 31(7), p. 277-286 (2009), in Russian.

14. T.A. Ignatyeva, A.N. Velikodnyi, A.A. San'kov, On the temperature dependence of the resistivity in Mo-Re, Mo-Re-Nb alloys // Voprosy atomnoi nauki tekhniki. Ser.: Vakuum, chistye materialy, sverkhprovodniki”, 14(6), p. 89-92 (2004), in Russian.

15. T.A. Ignatyeva, A.N. Velikodnyi, Peculiarities of the electron spectrum of alloys with the electrontopological transition and their influence on physical properties // Izvestiya RAN, ser. fiz. 71(8), p. 1104-1107 (2007), in Russian.

16. T.A. Ignatyeva, Peculiarities of the electron spectrum and temperature dependence of the molybdenum resistivity // Visnyk Kharkiv. Universytetu. Ser. fizychna: "Yadra, chastynky, polia", 763(1/33), p. 81-87 (2007), in Russian.

17. N.V. Volkenshtein, V.A. Novoselov, V.E. Startsev, Role of interelectron collisions in electroresistance // Zhurnal experimental. teoret. fiziki, 60(3), p. 1078 (1971), in Russian.

18. R.J. Iverson, L.Hodges, Molybdenum: band structure, Fermi surface and spin-orbit interaction // Phys. Rev. B, 8(4), p. 1429-1432 (1973).

19. I.M. Lifshits, On the structure of energy spectrum for impurity bands in disordered solid solutions // Zhurnal experimental. teoret. fiziki, 44, p. 1723 (1963), in Russian. 